\title{
A Study on the Cancer Adaptation, Family Support and Self-efficacy of Middle-aged Breast Cancer Patients
}

\author{
Hyesun Park1), Ryewon Ma2), Sunyoung Lim³)
}

\begin{abstract}
This study aims to identify cancer adaptation of breast cancer patients with high incidence in Korea and to analyze whether family support and self-efficacy affect. This correlational study was conducted among 75 middle-aged cancer patients with cancer living in the $\mathrm{S}$ and $\mathrm{K}$ regions in Korea. For data collection, a structured questionnaire was used for subjects who agreed to the study. The study tool measured breast cancer adaptation, family support, and self-efficacy and the collected data were analyzed using the SPSS / WIN 23.0 program. The general characteristics and degree of cancer adaptation, family support, and self-efficacy were analyzed using frequency and percentage, mean, and standard deviation, and the Pearson's coefficient was used for correlation between cancer adaptation, family support, and self-efficacy. The results showed that middle-aged breast cancer patients' cancer adaptation score was $77.03( \pm 6.79)$. The family support score was $14.56( \pm 13.28)$, and the self-efficacy was $40.15( \pm 12.26)$. The results show that, as family support and self-efficacy increased, the breast cancer adaptation result increased. Therefore, we suggest collecting basic data for the development of programs that can enhance cancer adaptation for middle-aged breast cancer patient.
\end{abstract}

Keywords: Cancer Adaptation, Family Support, Self-efficacy, Middle-aged Breast Cancer Patients

\section{Introduction}

Breast cancer in middle-aged women has a higher survival rate than those of other cancers, and long-term survival is achieved through early diagnosis and improved treatment levels[1]. In Korea, the incidence is high for ages 40-50 years or older[2]. Female breast cancer survivors experience long-term and late complications, and they often fail to solve various health problems[3]. Therefore, breast cancer survivors aged 50 and older are often chronically ill,

Received(November 23, 2019), Review Result(1st: December 27, 2019, 2nd: February 14, 2020), Accepted(April 25, 2020)

1) (Ph. D) 06974 Dept. of Red Cross College of Nursing, Chung-Ang University, 221 Heukseok-dong, Dongjak-gu, Seoul, Republic of Korea.

email: april1007@naver.com

2) (Assistant Professor, Corresponding Author) 51767 Dept. of Nursing, Kyungnam University, 7 Kyungnamdaehak-ro, Masanhappo-gu, Changwon-si, Gyeongsangnam-do, Republic of Korea.

email: mrw4023@Kyunganm.ac.kr

3) (Visiting Professor) 31065 Dept. of Nursing, Baekseok Culture University, 1 Baekseokdaehak-ro, Dongnam-gu, Cheonan-si, Chungcheongnam-do, Republic of Korea.

email: isokety@naver.com 
making physical and psychological adjustment important[2]. Cancer patients are often perceived to respond passively, such as when they exhibit feelings of despair and helplessness because they perceive the disease negatively. This requires intervention and management to alleviate the severity of the disease[4]. To improve the adaptation of cancer patients, efforts to improve the coping ability of these individuals are required. In a previous study on breast cancer patients who were receiving chemotherapy, the higher the family support, the higher the patients' psychosocial adaptation after surgery, chemotherapy, and radiation therapy. Previous studies have also shown that treatment does not return to its pre-treatment state even after the treatment is over, and it is reported that certain after effects, such as depression and anxiety, make adaptation difficult[5].

Support for middle-aged women increases social adaptation and helps them overcome disease; in particular, family support is known to increase the psychological and social adaptation of breast cancer patients and affect health problems[6]. In addition, it was found that, the higher the family support, the less pain the cancer patient had and the more positively they responded to treatment, which promotes healthy behavior[7].

Self-efficacy, which is an individual's belief and confidence that they can achieve the results that they want by adjusting their behavior and having confidence in themselves, synchronizes itself in an unpredictable situation[8]. Self-efficacy in middle-aged women has been shown to control symptoms of breast cancer, improve cancer treatment performance, and have a positive effect on quality of life as well as coping strategies[9].

This study aims to identify the degree of cancer adaptation, family support, and self-efficacy found in middle-aged female cancer patients, analyze the relationship between the variables, and present basic data for the development of programs to promote the cancer adaptation of middle-aged breast cancer patients in the future.

\section{Methods}

\subsection{Study Design}

This descriptive correlation study aims to identify the cancer adaptation, family support, and self-efficacy, of and examine correlations among variables in middle-aged breast cancer patients.

\subsection{Sample and Data Collection}


This study surveyed 75 breast cancer patients aged 50 and older who were from the $S$ and $K$ regions in Korea. After explaining the purpose of the study and how to complete the questionnaire, the survey was distributed to the subjects who after decided to participate and gave distribute written consent. The number of subjects studied was calculated using G*power3.1.3. Based on the prior study[10], 68 people were subject to the least needed study based on the significance level of 0.05 and the power of 0.8 and the effect size of 0.15 respectively

\subsection{Assessment Instruments}

\subsubsection{Cancer Adaptation}

The Self-Adjustment to Cancer tool, which is a self-assessment that evaluates how people cope with cancer[11] was measured here by using the Mini-Mental Adjustment to Cancer tool, which included 29 questions that were corrected and supplemented[12]. There were 5 measures, including helplessness hopelessness $(\mathrm{HH})$, anxious preoccupation (AP), fatalism (FA), fighting spirit (FS), and cognitive avoidance (CA). The Korean version is composed of four sub-areas: Helpless-Hopeless, anxiety, cognitive avoidance, and positive attitude. The positive attitude is a combination of the fatalism and the fighting spirit acceptance of the original tool. This tool was composed of 5 sub-categories, which were each rated on a Likert scale, from 1 ("not at all") to 4 ("very good"). The Cronbach's alpha was .734.

\subsubsection{Family Support}

Family support, which is a measure of social support that is perceived by individuals, was developed by Zimet et al.[13]. Among the tools developed in Korean, scores that were calculated for the areas constituting family support were measured via a Likert scale, from 1 ("not at all") to 5 ("very true"). The Cronbach's alpha was .921.

\subsubsection{Self-Efficacy}

The Self-Efficacy Scale for Self-Management of Breast Cancer patients was used to measure self-efficacy in breast patients[14]. It consists of 5 subscales of 13 questions to measure self-efficacy in coping with psychological information needs, managing side effects, maintaining 
a healthy lifestyle, implementing treatment, and having sex. The Cronbach's alpha was .982.

\subsection{Analysis}

The collected data were analyzed using the SPSS / WIN 23.0 program. The general characteristics and degree of cancer adaptation, family support, and self-efficacy were analyzed using frequency and percentage, mean, and standard deviation, and the Pearson's coefficient was used for correlation between cancer adaptation, family support, and self-efficacy.

\subsection{Ethical Considerations}

The purpose of the study was explained to all participants, who were informed that their involvement was voluntary. Participants were assured that the information they provided would remain confidential.

\section{Results}

\subsection{General Characteristics}

The average age of the subjects was 56.6 years old, with 58 married (77.3\%) and 17 unmarried (22.7\%). Religion affiliations were as follows: 32 Christians (42.7\%), 8 Buddhists (10.6\%), 8 Catholics $(10.7 \%)$, and 27 non-religious (36.0\%). Regarding education, there was 1 graduate from middle school (1.3\%), 15 from high school (20.0\%), 51 university graduates $(68.0 \%)$, and 8 graduate students $(10.7 \%)$. The stages of cancer included $32(42.7 \%)$ in stage 1, $29(38.6 \%)$ in stage 2, $11(14.7 \%)$ in stage 3, and $3(4.0 \%)$ in stage 4 , with 23 patients who were completely resected as a surgical type (30.7\%) and 52 who had partial resections $(69.3 \%)$.

Among the general characteristics, the most prominent part was married women, college graduates, and first-stage cancer patients [Table 1].

[Table 1] General Characteristics

$(\mathrm{n}=75)$

\begin{tabular}{llll}
\hline \multicolumn{1}{c}{ Variables } & \multicolumn{1}{c}{ Categories } & $\mathrm{n}(\%)$ & Mean(SD) \\
\hline Age & & & $56.6( \pm 3.41)$ \\
\hline Marriage & Unmarried & $58(77.3 \%)$ & \\
& Married & $17(22.7 \%)$ & \\
\hline Religion & Christians & $32(42.7 \%)$ &
\end{tabular}




\begin{tabular}{llc} 
& & http://dx.doi.org/10.21742/apjcri.2020.05.08 \\
& Buddhists & $8(10.6 \%)$ \\
& Catholics & $8(10.7 \%)$ \\
Education & mon-religious & $27(36.0 \%)$ \\
& hiddle school school & $1(1.3 \%)$ \\
& university graduates & $15(20.0 \%)$ \\
& graduate students & $51(68.0 \%)$ \\
Cancer stage & 1 period & $8(10.7 \%)$ \\
& 2 period & $32(42.7 \%)$ \\
& 3 period & $29(38.6 \%)$ \\
& 4 period & $11(14.7 \%)$ \\
\hline Surgical type & completely resection & $3(4.0 \%)$ \\
& partial resection & $23(30.7 \%)$ \\
& & $52(69.3 \%)$ \\
\hline
\end{tabular}

\subsection{Cancer Adaptation and Family Support, Self Efficacy}

The average cancer adaptation level of the study subjects was 77.03 (6.79), with the sub-variables as follows: 26.17 (3.12) for helplessness-hopelessness, 19.53 (2.83) for anxiety, 12.16 (2.84) for fate, 12.68 (2.70) for fighting spirit, and 8.94 (1.93) for cognitive avoidance. Family support was 14.56 (3.30), while self-efficacy was 40.15 (12.26).

In this study, the degree of cancer adaptation was high in the Helpless-Hopeless, Anxious Preoccupation, above average and the fatalism and fighting spirit were low in the level [Table 2].

[Table 2] Cancer Adaptation, Family Support, and Self-Efficacy of Subjects

$(n=75)$

\begin{tabular}{llccc}
\hline \multicolumn{1}{c}{ Variables } & & $\begin{array}{c}\text { M(SD) } \\
\text { number of items }\end{array}$ & Min & Max \\
\hline Cancer Adaptation & Total & $77.03(6.79)$ & 63 & 96 \\
& Helpless-Hopeless & $26.17(3.12)$ & 20 & 32 \\
& Anxious Preoccupation & $19.53(2.83)$ & 11 & 26 \\
& Fatalism & $12.16(2.84)$ & 7 & 19 \\
& Fighting Spirit & $12.68(2.70)$ & 8 & 19 \\
& Cognitive Avoidance & $8.94(1.93)$ & 4 & 13 \\
\hline Family Support & & $14.56(3.30)$ & 8 & 20 \\
\hline Self Efficacy & & $40.15(12.26)$ & 17 & 60 \\
\hline
\end{tabular}

\subsection{Relationship between Cancer Adaptation, Family Support and Self Efficacy}

The results found that, the higher the family support and self-efficacy, the higher the cancer 
adaptation. Cancer adaptation, family support, and self-efficacy were found to have a significant correlation [Table 3].

[Table 3] Relationship between Cancer Adaptation, Family Support and Self-Efficacy

$(\mathrm{n}=75)$

\begin{tabular}{cccc}
\hline & Cancer Adaptation & Family Support & Self Efficacy \\
\hline Cancer Adaptation & 1 & $.587^{* *}$ & $.630^{* *}$ \\
\hline Family Support & & 1 & $.851^{* *}$ \\
\hline Self Efficacy & & & 1 \\
\hline${ }^{* *} \mathrm{p}<.001$ & &
\end{tabular}

\section{Discussion}

This study was conducted to identify the cancer adaptation, family support, and self-efficacy levels of middle-aged female breast cancer patients and to investigate the correlation between variables and factors that affect cancer adaptation.

In this study, the average cancer adaption of middle-aged women was 26.17 for helplessness - hopelessness, 19.53 for anxious preoccupation, 12.16 for fatalism, 12.68 for fighting spirit, and 8.94 for cognitive avoidance. Helplessness - hopelessness means that you think you can't control the disease yourself, and give up. Anxious Preoccupation is anxiety and anxiety about the recurrence or worsening of cancer, and cognitive avoidance means turning your attention to not thinking about the disease. Fatalism and fighting Spirit are areas of positive adaptation. In a previous study[15] on middle-aged men with lung cancer, the results were 13.82 for helplessness - hopelessness, 19.54 for anxious preoccupation, 13.83 for fatalism, 11.97 for fighting spirit, and 10.38 for cognitive avoidance when using the same tool.

In another previous study[10] on patients with a malignant bone tumor, the results found 12.84 for helplessness-hopelessness, 18.99 for anxious preoccupation, 10.59 for cognitive avoidance and 25.15 for positive attitude. In this study about cancer adaptation of the patient, helplessness-hopelessness and anxious preoccupation was higher than in the previous study and cognitive avoidance was lower than in the previous study[10]. These differences were likely found because the study on lung cancer included male patients aged 45-64 years who were receiving both outpatient and inpatient treatment at a third-generation hospital[15], and in the research on malignant bone tumors, the average age was 39 years, with males accounting for $52 \%$ of the sample[10]. Therefore, the current study is considered different due to its subjects being middle-aged female breast cancer patients. 
In previous studies by Kornblith et al.[16] and Kim and Ko[17], younger women with breast cancer scored higher for psychological pain than did older women with breast cancer. However, it was also found that, the younger the age, the higher the degree of adaptation to anticancer drugs[18]. In addition, if the patient's age was relatively higher, they were often receiving complex treatment, including combined therapy, such as surgery, chemotherapy, and radiation therapy. Therefore, this may explain why, among the cancer adaptation sub-variables, helplessness - hopelessness and anxious preoccupation was higher than it was for male patients with lung cancer. Efforts to improve the cancer adaptation of cancer patients in middle-aged women are needed, and in particular, interventions are needed to reduce the feeling of helplessness and anxiety[19]. In addition, efforts are needed to increase the positive item Fatalism and fighting Spirit.

Based on the above, an effort to increase cancer adaptation for middle-aged women with cancer is required. An arbitration method is especially needed to lower feelings of helplessness - hopelessness.

In this study, there was a significant positive correlation between cancer adaptation, family support, and cancer adaptation and self-efficacy. Thus, if family support and self-efficacy are higher, the degree of cancer adaptation is higher. Social support was shown to affect cancer adaptation in a previous study on thyroid cancer[20], while the current study reflects that cancer adaptation is positively correlated with family support and its amount. In a study by Park et al.[21], the grade of anxiety and depression was significantly higher in female patients than it was in male patients, and they found that emotional temperament could cause emotional disorders. In addition, in previous studies[22] on patients who had suffered with breast cancer for 10 years, it was found that high helplessness-hopelessness scores had a negative effect on survival rate.

Self-efficacy is known as an important variable for predicting health behavior through active methods[8]; a previous study[23] reported that self-efficacy positively affects health behavior, symptom control, and treatment compliance in breast cancer. In another study of breast cancer patients[24], when they applied a self-efficacy incensement program, the patients' physical symptoms decreased and self-care performance improved. Therefore, it is necessary to develop and operate an intervention program that could increase the cancer adaptation and self-efficacy of as well as instill realistic confidence in middle-aged female breast cancer patients.

In this study, family support showed a significant correlation between cancer adaptation and self-efficacy, which coincides with the result that the higher family support, the higher the cancer adaptation in a study by Kim et al[25]. It is also similar to a result in a previous study 
that found the higher the level of support for the female breast cancer patients spouse, the higher the level of psychological adaptation of breast cancer patients or those with other cancers[26][27].

Family support is important because it helps patients adapt to change more than any other form of social support; it is well known that, the more positive the family relationship, the better is the psychosocial adaptation[25][28][29].

Thus, to increase the cancer adaptation of middle-aged female breast cancer patients, intervention development is required to improve self-efficacy, increase family support, and increase patients' confidence.

This study was meaningful in that it perceived the degree of cancer adaptation of middle-aged female breast cancer patients and identified related factors. The systematic results regarding the influencing factors will be used as the basis for an intervention program.

The limitations of this study are that the results of a study conducted on breast cancer patients in middle-aged women have been released, so care needs to be taken to generalize various middle-aged female cancer patients.

\section{Conclusion}

This study was attempted to obtain basic data for the development of cancer adaptation program for middle-aged female cancer patients to determine whether family support and self-efficacy affect cancer adaptation. This research shows a significant positive correlation among cancer adaptation, family support, and self-efficacy. The higher the family support and self-efficacy, the better the cancer adaptation. Based on the above results, future studies should continue to cancer adaptation for middle-aged female cancer patients. It is suggested that middle-aged female cancer patients should be used as basic data for counseling and program development that can improve their family support and self-efficacy. In addition, it is suggested that further research is needed to identify the impact factors of cancer adaptation in consideration of various variables other than demographic and disease-related variables that can affect cancer adaptation in middle-aged female cancer patients.

\section{Reference}

[1] H. Park, Breast Cancer Facts \& Figures, Korean Journal of Clinical Oncology, (2018) 
[2] Y. K. Oh, S. Y. Hwang, Impact of Uncertainty on the Quality of Life of Young Breast Cancer Patients: Focusing on Mediating Effect of Marital Intimacy, Journal of Korean Academy of Nursing, (2018), Vol.48, No.1, pp.50-58. DOI: 10.4040/jkan.2018.48.1.50

[3] J. A. Lee, J. H. Yu, Y. M. Song, Management of long-term breast cancer survivors in Korea, Journal of the Korean Medical Association, (2016), Vol.59, No.4, pp.266-275. DOI: 10.5124/jkma.2016.59.4.266

[4] P. Hopman, M. Rijken, Illness perceptions of cancer patients: relationships with illness characteristics and coping, Psycho-Oncology, (2015), Vol.24, No.1, pp.11-18. DOI: 10.1002/pon.3591

[5] Y. J. Kim, E. J. Yang, Psychological Systematic Consideration of Breast Cancer Radiotherapy, Journal of the Korean Society of Radiology, (2019), Vol.13 No.4, pp.629-635. DOI: 10.7742/jksr.2019.13.4.629

[6] J. Seo, Construction of a Model for Psychosocial Adjustment of Breast Cancer Survivors Based on Family Resilience, Seoul National University, Ph. D Thesis, (2018)

[7] K. S. Jeong, J. E. Heo, Y. S. Tea, Relationships among Distress, Family Support, and Health Promotion Behavior in Breast Cancer Survivors, Asian Oncology Nursing, (2014), Vol.14, No.3, pp.146-154. UCI: G704-SER000008675.2014.14.3.003

[8] A. Bandura, Self-efficacy in changing societies, Cambridge University Press, (1995). DOI: 10.1017/CBO9780511527692

[9] S. Akin, G. Can, Z. Durna, A. Aydiner, The quality of life and self-efficacy of Turkish breast cancer patients undergoing chemotherapy, European Journal of Oncology Nursing, (2008), Vol.12, No.5, pp.449-456. DOI: $10.1016 /$ j.ejon.2008.07.006

[10] Y. Yoon, A Study on Illness Perception, Family Hardiness and Mental Adjustment to Cancer of Primary Malignant Bone Tumor Patients, The Graduate School Ewha Womans University, Master Thesis, (2017)

[11] M. Watson, S. Greer, J. Young, Q. Inayat, C. Burgess, B. Robertson, Development of a questionnaire measure of adjustment to cancer: the MAC scale, Psychological Medicine, (1988), Vol.18, No.1, pp.203-209. DOI: $10.1017 / \mathrm{S} 0033291700002026$

[12] J. I. Kang, H. C. Chung, S. J. Kim, H. J. Choi, J. B. Ahn, H. C. Jeung, K. Namkoong, Standardization of the Korean version of Mini-Mental Adjustment to Cancer (K-Mini-MAC) scale: factor structure, reliability, and validity, Psycho-Oncology, (2008), Vol.17, No.6, pp.592-597. DOI: 10.1002/pon.1277

[13] Zimet, G. D., Dahlem, N. W., Zimet, S. G., Farley, G. K., The multidimensional scale of perceived social support, Journal of Personality Assessment, (1988), Vol.52, No.1, pp.30-41. DOI: 10.1207/S15327752JPA5201_2

[14] R. Lee, S. H. Kim, K. S. Lee, M. K. Seo, Development and Validation of Self-Efficacy Scale for Self-Management of Breast Cancer (SESSM-B), Journal of Korean Academy Nursing, (2012), Vol.42, No.3, pp.385-395. DOI: 10.4040/jkan.2012.42.3.385

[15] Y. Yoo, Stigma, Adjustment, and Quality of Life of Middle-aged Male Patients with Lung Cancer, Seoul National University, Ph. D Thesis, (2018)

[16] Kornblith A. B, Powell M, Regan M. M, Bennett S, Krasner C, Moy B, Younger J, Goodman A, Berkowitz R, Winer E, Long-term psychosocial adjustment of older vs younger survivors of breast and 
endometrial cancer, Psycho-oncology, (2007), Vol.16, No.10, pp.895-903. DOI: 10.1002/pon.1146

[17] H. Y. Kim, E. Ko, Psychosocial Adjustment between Younger and Older Breast Cancer Survivors, Asian Oncology Nursing, (2012), Vol.12, No.4, pp.280-288. DOI: 10.5388/aon.2012.12.4.280

[18] S. Kim, Uncertainty, Coping, and Adjustment of Cancer Patients Participating in Clinical Trials of Anticancer Drugs, The Graduate School Seoul National University, Master Thesis, (2018)

[19] S. Lee, Y. J. Kim, Y. J. Choi, Does she advance her development in the face of cancer? A structural equation model of post-traumatic growth after diagnosed with cancer, International Journal of Advanced Nursing Education and Research, (2018), Vol.3, No.2, pp.1-10.

[20] S. Y. Lee, H. K. Kim, Structural Equation Modeling on Health-related Quality of Life among Patients with Thyroid Cancer, Korean Journal of Adult Nursing, (2018), Vol.30 No.2, pp.171-182. DOI: 10.7475/kjan.2018.30.2.171

[21] S. Y. Park, S. J. Kim, J. I. Kang, Characteristics of Affective Temperament and Vulnerability of Depression in Young Women, Journal of Society of Korean Women Psychiatrists, (2015), Vol.13, No.1, pp.12-18.

[22] Watson M, Homewood J, Haviland J, Bliss J. M, Influence of psychological response on breast cancer survival: 10-year follow-up of a population-based cohort, Eurropean Journal Cancer, (2005), Vol.41, No.12, pp.1710-1714. DOI: 10.1016/j.ejca.2005.01.012

[23] S. N. Aikman, S. Doyle-Portillo, P. Verhaeghen, N. Simmons, The Effect of Instruction Point of View on Self-Efficacy for Performing Breast Self-Exams, American Journal of Health Education, (2017), Vol.48, No.1, pp.1-10. DOI: 10.1080/19325037.2016.1250687

[24] S. Y. Liang, T. C. Chao, L. M. Tseng, S. L. Tsay, K. C. Lin, H. H. Tung, Symptom-Management Self-Efficacy Mediates the Effects of Symptom Distress on the Quality of Life among Taiwanese Oncology Outpatients with Breast Cancer, Cancer Nursing, (2016), Vol.39, No.1, pp.67-73. DOI: 10.1097/NCC.0000000000000244

[25] H. S. Kim, M. H. Lee, H. Y. Kim, J. H. Nho, Factors Affecting Psychosocial Adjustment in Patients with Surgical Removal of Benign Breast Tumor, Korean Journal of Women Health Nursing, (2018), Vol.24 No.2, pp.163-173. DOI: $10.4069 /$ kjwhn.2018.24.2.163

[26] Y. Seo, Construction of a Model for Psychosocial Adjustment of Breast Cancer Survivors Based on Family Resilience, Seoul National University, Ph. D Thesis, (2018).

[27] J. H. Moon, Marital Satisfaction, Marital Intimacy, Spouse Support, Physical Symptom and Wellbeing with Breast Cancer Patient, Journal of The Korean Data Analysis Society, (2016), Vol.18, No.2, pp.937-950. UCI: G704-000930.2016.18.2.036

[28] E. Y. Noh, H. Y. Kwon. The Mediating Effect of Meaning of Life and Savoring Belief in the Relationship between Family Strength and Psychological Well-being in Middle-Aged Women, Asia-pacific Journal of Psychology and Counseling, (2018), Vol.2, No.2, pp.105-110. DOI: 10.21742/apjpc.2018.2.2.18

[29] Y. H. Kim, S. J. Cho, The Effect of Middle-Aged Women's Ego Resilience on Family Adaptability, Asia-pacific Journal of Psychology and Counseling, (2019), Vol.3, No.1, pp.13-18, DOI: 10.21742/apjpc.2019.3.1.03 